\title{
ON LIE-LIKE COMPLEX FILIFORM LEIBNIZ ALGEBRAS
}

\author{
B. A. OMIROV ${ }^{\bowtie}$ and I. S. RAKHIMOV
}

(Received 16 June 2008)

\begin{abstract}
In this paper we propose an approach to classifying a subclass of filiform Leibniz algebras. This subclass arises from the naturally graded filiform Lie algebras. We reconcile and simplify the structure constants of such a class. In the arbitrary fixed dimension case an effective algorithm to control the behavior of the structure constants under adapted transformations of basis is presented. In one particular case, the precise formulas for less than 10 dimensions are given. We provide a computer program in Maple that can be used in computations as well.
\end{abstract}

2000 Mathematics subject classification: primary 17A32, 17B30; secondary 17A60, $17 \mathrm{~B} 70$.

Keywords and phrases: Lie algebra, filiform Leibniz algebra, natural gradation, isomorphism criterion.

\section{Introduction}

It is well known that the natural gradation of nilpotent Lie and Leibniz algebras is very helpful in investigating their structural properties. This technique is more effective when the length of the natural gradation is sufficiently large. When it is maximal the algebra is called filiform. For applications of this technique, see [6, 12] (for Lie algebras) and [1] (for Leibniz algebras).

The present paper deals with the filiform Leibniz algebras whose natural gradation is a filiform Lie algebra. Note that the case of algebras whose natural gradation is a non-Lie filiform Leibniz algebra was considered in [1-3, 5].

Section 2 gathers together basic definitions, notation and conventions used in this paper. Section 3 is devoted to the description of the class of non-Lie filiform Leibniz algebras whose gradation is a filiform Lie algebra. It contains the main result of the paper. Here we simplify the multiplication table and follow the behavior of the structure constants under the adapted action. Section 4 deals with the detailed description of one particular case. In this section we give precise formulas for changing of the structure constants and a simple computer program in Maple.

This work was supported by the Science Fund Grant Project 06-01-04-SF0122 MOSTI (Malaysia).

(c) 2009 Australian Mathematical Society 0004-9727/2009 \$16.00 


\section{Preliminaries}

Let $V$ be a vector space of dimension $n$ over an algebraically closed field $K$ (char $K=0$ ). Bilinear maps $V \times V \rightarrow V$ form a vector space $\operatorname{Hom}(V \otimes V, V)$ of dimension $n^{3}$, which can be considered together with its natural structure of an affine algebraic variety over $K$ and denoted by $\operatorname{Alg}_{n}(K) \cong K^{n^{3}}$. An $n$-dimensional algebra $L$ over $K$ can be considered as an element $\lambda(L)$ of $\operatorname{Alg}_{n}(K)$ via the bilinear mapping $\lambda: L \otimes L \rightarrow L$ defining a binary algebraic operation on $L:$ let $\left\{e_{1}, e_{2}, \ldots, e_{n}\right\}$ be a basis of the algebra $L$. Then the multiplication table of $L$ is represented by point $\left(\gamma_{i j}^{k}\right)$ of this affine space as follows:

$$
\lambda\left(e_{i}, e_{j}\right)=\sum_{k=1}^{n} \gamma_{i j}^{k} e_{k}
$$

Here $\gamma_{i j}^{k}$ are called structure constants of $L$. The linear reductive group $G L_{n}(K)$ acts on $\operatorname{Alg}_{n}(K)$ by $(g * \lambda)(x, y)=g\left(\lambda\left(g^{-1}(x), g^{-1}(y)\right)\right)$ ('transport of structure'). Two algebras $\lambda_{1}$ and $\lambda_{2}$ are isomorphic if and only if they belong to the same orbit under this action. It is clear that the elements of the given orbit are algebras isomorphic to each other.

Recall that an algebra $L$ over a field $F$ is called a Leibniz algebra if its composition law $[\cdot, \cdot]$ satisfies the following so-called Leibniz identity:

$$
[x,[y, z]]=[[x, y], z]-[[x, z], y] .
$$

Leibniz algebras were introduced by Loday [8, 9]. (For this reason, they have also been called 'Loday algebras' [7].) A skew-symmetric Leibniz algebra is a Lie algebra. In this case (2.1) is just the Jacobi identity.

Let $L B_{n}(K)$ be a subvariety of $\operatorname{Alg}_{n}(K)$ consisting of all $n$-dimensional Leibniz algebras over $K$. It is invariant under the above-mentioned action of $G L_{n}(K)$. As a subset of $\operatorname{Alg}_{n}(K)$ the set $L B_{n}(K)$ is specified by the system of equations with respect to the structure constants $\gamma_{i j}^{k}$ :

$$
\sum_{l=1}^{n}\left(\gamma_{j k}^{l} \gamma_{i l}^{m}-\gamma_{i j}^{l} \gamma_{l k}^{m}+\gamma_{i k}^{l} \gamma_{l j}^{m}\right)=0 .
$$

In fact, theoretically, by solving the above system of equations we can get a complete classification of Leibniz algebras in a given dimension $n$. But in practice this approach is useless even for small values of $n$. Therefore one usually has to apply different methods of investigation.

If $L$ is a Leibniz algebra we may define

$$
L^{1}=L, \quad L^{k+1}=\left[L^{k}, L\right], \quad k \geq 1 .
$$

The series

$$
L^{1} \supseteq L^{2} \supseteq L^{3} \supseteq \cdots
$$


is called the descending central series of $L$. If the series terminates for some positive $n$, then the Leibniz algebra $L$ is said to be nilpotent. The termination number here is called the nilindex of $L$. Below we present a gradation closely related to the descending central series.

Given a nilpotent Leibniz algebra $L$ with nilindex $s$, we put $L_{i}=L^{i} / L^{i+1}, 1 \leq i \leq$ $s-1$, and $g r L=L_{1} \oplus L_{2} \oplus \cdots \oplus L_{s-1}$. Since $\left[L_{i}, L_{j}\right] \subseteq L_{i+j}$ the algebra $g r L$ is graded. $g r L$ is called the naturally graded Leibniz algebra.

Let us introduce the class of nilpotent Leibniz algebras, which is main object of the paper. In the case of Lie algebras, such algebras have the maximal nilindex.

Definition 2.1. A Leibniz algebra $L$ is said to be filiform if $\operatorname{dim} L^{i}=n-i$, where $n=\operatorname{dim} L$ and $2 \leq i \leq n$.

Clearly a filiform Leibniz algebra is nilpotent.

Let Leib $_{n}$ denote the class of all $n$-dimensional filiform Leibniz algebras.

DEFINITION 2.2. If a Leibniz algebra $G$ is isomorphic to a filiform naturally graded algebra $g r L$, then $G$ is said to be naturally graded filiform Leibniz algebra.

Later on all algebras are supposed to be over the field of complex numbers $\mathbb{C}$ and the omitted products of basis vectors are supposed to be zero.

The following theorem summarizes the results of $[1,12]$.

THEOREM 2.3. Any complex $(n+1)$-dimensional naturally graded filiform Leibniz algebra is isomorphic to one of the following pairwise nonisomorphic algebras:

$$
\begin{aligned}
& N G F_{1}=\left\{\begin{array}{l}
{\left[e_{0}, e_{0}\right]=e_{2},} \\
{\left[e_{i}, e_{0}\right]=e_{i+1}, \quad 1 \leq i \leq n-1,}
\end{array}\right. \\
& N G F_{2}= \begin{cases}{\left[e_{0}, e_{0}\right]=e_{2},} \\
{\left[e_{i}, e_{0}\right]=e_{i+1}, \quad 2 \leq i \leq n-1,}\end{cases} \\
& N G F_{3}= \begin{cases}{\left[e_{i}, e_{0}\right]=-\left[e_{0}, e_{i}\right]=e_{i+1},} & 1 \leq i \leq n-1, \\
{\left[e_{i}, e_{n-i}\right]=-\left[e_{n-i}, e_{i}\right]=\alpha(-1)^{i+1} e_{n},} & 1 \leq i \leq n-1 .\end{cases}
\end{aligned}
$$

$\alpha \in\{0,1\}$ for odd $n$ and $\alpha=0$ for even $n$.

It is clear that neither $N G F_{1}$ nor $N G F_{2}$ is a Lie algebra.

The above theorem means that the natural gradation of a Leibniz algebra is in one of the classes $N G F_{i}$ for $i=1,2,3$.

The following result of [1] describes the class of complex filiform Leibniz algebras whose natural gradation is one of $N G F_{i}$ for $i=1,2$.

THEOREM 2.4. Any $(n+1)$-dimensional complex non-Lie filiform Leibniz algebra, whose naturally graded algebra is not a Lie algebra, belongs to one of the following two classes: 


$$
\begin{aligned}
& F \text { Leib }_{n+1}= \begin{cases}{\left[e_{0}, e_{0}\right]=e_{2},} & \\
{\left[e_{i}, e_{0}\right]=e_{i+1},} & 1 \leq i \leq n-1, \\
{\left[e_{0}, e_{1}\right]=\alpha_{3} e_{3}+\alpha_{4} e_{4}+\cdots+\alpha_{n-1} e_{n-1}+\theta e_{n},} & \\
{\left[e_{j}, e_{1}\right]=\alpha_{3} e_{j+2}+\alpha_{4} e_{j+3}+\cdots+\alpha_{n+1-j} e_{n},} & 1 \leq j \leq n-2, \\
\alpha_{3}, \alpha_{4}, \ldots, \alpha_{n}, \theta \in \mathbb{C} ; & \end{cases} \\
& \operatorname{SLeib}_{n+1}= \begin{cases}{\left[e_{0}, e_{0}\right]=e_{2},} & \\
{\left[e_{i}, e_{0}\right]=e_{i+1},} & 2 \leq i \leq n-1, \\
{\left[e_{0}, e_{1}\right]=\beta_{3} e_{3}+\beta_{4} e_{4}+\cdots+\beta_{n} e_{n},} & \\
{\left[e_{1}, e_{1}\right]=\gamma e_{n},} & \\
{\left[e_{j}, e_{1}\right]=\beta_{3} e_{j+2}+\beta_{4} e_{j+3}+\cdots+\beta_{n+1-j} e_{n},} & 2 \leq j \leq n-2, \\
& \beta_{3}, \beta_{4}, \ldots, \beta_{n}, \gamma \in \mathbb{C} .\end{cases}
\end{aligned}
$$

Isomorphism criteria, classifications and invariants of $F$ Leib $_{n+1}$ and $S \operatorname{Leib}_{n+1}$ were investigated in $[2,3,5,10,11]$.

\section{Main result}

This section is devoted to the filiform Leibniz algebras whose natural gradation is an algebra from $N G F_{3}$. This class is denoted by $T$ Leib $_{n+1}$. Here we clarify the multiplication table of algebras from $T$ Leib $_{n+1}$ and investigate the behavior of structure constants under a change of basis.

PROPOSITION 3.1.

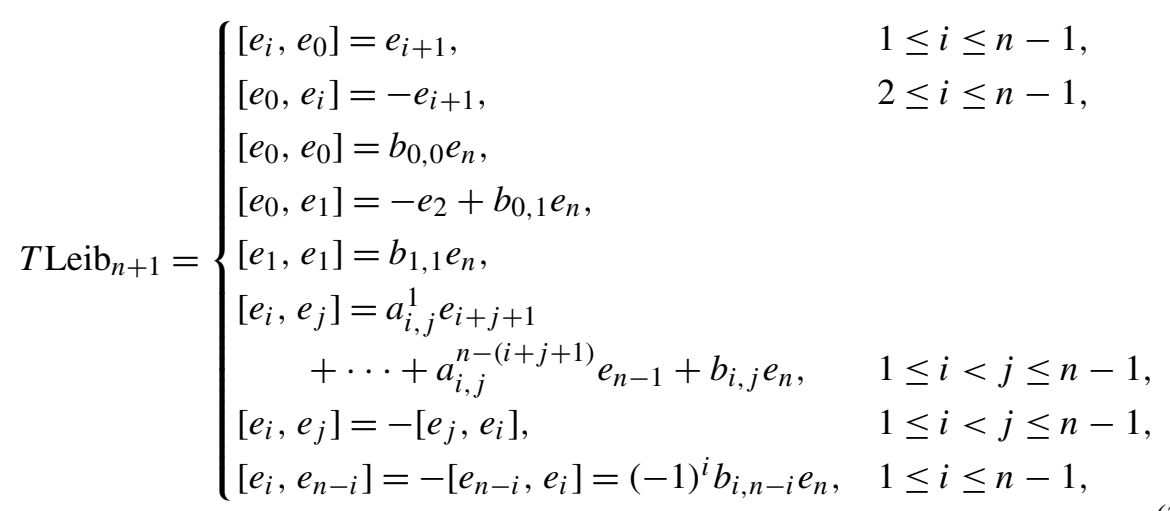

where $a_{i, j}^{k}, b_{i, j} \in \mathbb{C}$ and $b_{i, n-i}=b$ whenever $1 \leq i \leq n-1, b \in\{0,1\}$ for odd $n$ and $b=0$ for even $n$.

Proof. Let $L \in T \operatorname{Leib}_{n+1}$ and $\left\{e_{0}, e_{1}, \ldots, e_{n}\right\}$ be a basis of $L$. Then it is clear that $\left[e_{i}, e_{j}\right] \in\left\langle e_{i+j+1}, \ldots, e_{n}\right\rangle$ for any $i, j \neq 0$. Then

$$
\left[e_{i}, e_{0}\right]=e_{i+1}+(*) e_{i+2}+\cdots+(*) e_{n}, \quad 1 \leq i \leq n-1 .
$$


Putting $e_{1}^{\prime}=e_{1}, e_{0}^{\prime}=e_{0}, e_{i+1}^{\prime}:=\left[e_{i}^{\prime}, e_{0}^{\prime}\right]$, we can assume that $\left[e_{i}, e_{0}\right]=e_{i+1}, 1 \leq i \leq$ $n-1$.

Now consider

$$
\left[e_{0}, e_{i}\right]=-e_{i+1}+\alpha_{0, i}^{i+2} e_{i+2}+\alpha_{0, i}^{i+3} e_{i+3}+\cdots+\alpha_{0, i}^{n} e_{n}, \quad 1 \leq i \leq n-1 .
$$

We get

$$
\left[e_{i}, e_{0}\right]+\left[e_{0}, e_{i}\right]=\alpha_{0, i}^{i+2} e_{i+2}+\alpha_{0, i}^{i+3} e_{i+3}+\cdots+\alpha_{0, i}^{n} e_{n}, \quad 1 \leq i \leq n-1 .
$$

Note that from the Leibniz identity it follows that $[x, y]+[y, x] \in \Re(L)$, for any $x, y \in L$, where $\mathfrak{R}(L)$ is the right annihilator of $L$. Therefore, if we multiply the both sides of (3.2) from the left-hand side $(n-i-2)$ times by $e_{0}$ we obtain $\alpha_{0, i}^{i+2}=0$. Substituting and repeating, we get

$$
\alpha_{0, i}^{i+k}=0, \quad 2 \leq k \leq n-1-i .
$$

Applying the above to $\left[e_{i}, e_{i}\right], 0 \leq i \leq[n / 2]$, we get $\left[e_{i}, e_{i}\right]=\alpha_{i, i}^{n} e_{n}$.

The chain of equalities

$$
\begin{aligned}
{\left[e_{0}, e_{i}\right] } & =\left[e_{0},\left[e_{i-1}, e_{0}\right]\right]=\left[\left[e_{0}, e_{i-1}\right], e_{0}\right]-\left[\left[e_{0}, e_{0}\right], e_{i-1}\right] \\
& =\left[-e_{i}+\alpha_{0, i-1}^{n} e_{n}, e_{0}\right]=-\left[e_{i}, e_{0}\right]=-e_{i+1}
\end{aligned}
$$

leads to $\left[e_{i}, e_{0}\right]=-\left[e_{0}, e_{i}\right]=e_{i+1}$ for $2 \leq i \leq n-1$, that is, $\left[e_{0}, x\right]=-\left[x, e_{0}\right]$ for any $x \in L^{2}$. We claim that

$$
\left[e_{i}, e_{j}\right]=-\left[e_{j}, e_{i}\right], \quad 1 \leq i<j \leq n .
$$

The induction argument by $i$ for any $j$ and the chain of equalities

$$
\begin{aligned}
{\left[e_{i}, e_{j+1}\right] } & =\left[e_{i},\left[e_{j}, e_{0}\right]\right]=\left[\left[e_{i}, e_{j}\right], e_{0}\right]-\left[\left[e_{i}, e_{0}\right], e_{j}\right] \quad\left(\operatorname{since}\left[e_{i}, e_{j}\right] \in L^{2}\right) \\
& =-\left[e_{0},\left[e_{i}, e_{j}\right]\right]+\left[\left[e_{0}, e_{i}\right]-\alpha_{0, i}^{n} e_{n}, e_{j}\right]=-\left[e_{0},\left[e_{i}, e_{j}\right]\right]+\left[\left[e_{0}, e_{i}\right], e_{j}\right] \\
& =-\left[\left[e_{0}, e_{i}\right], e_{j}\right]+\left[\left[e_{0}, e_{j}\right], e_{i}\right]+\left[\left[e_{0}, e_{i}\right], e_{j}\right] \\
& =-\left[e_{j+1}, e_{i}\right], \quad 1 \leq j \leq n-1,
\end{aligned}
$$

show (3.3).

The above observations lead to the required multiplication table of $L \in$ $T$ Leib $_{n+1}$.

Note that filiform Lie algebras are in TLeib.

Bearing in mind Theorem 2.4 and all the foregoing, we conclude that the set of all $n$-dimensional filiform Leibniz algebras can be represented as a disjoint union of $F$ Leib $_{n}, S$ Leib $_{n}$ and $T$ Leib $_{n}$.

Let $L \in T$ Leib $_{n+1}$. Then it is easy to see that the subspace spanned by $\left\{e_{n}\right\}$ is an ideal of $L$ and the quotient algebra $L /\left\langle e_{n}\right\rangle$ is an $n$-dimensional filiform Lie algebra. Therefore, later on in this case the structure constants $a_{i, j}^{k}$ will be considered to be satisfying the Leibniz identity. Moreover, $n$-dimensional filiform Lie algebras have been classified (see [4]). In order to make use of the existing classification of $n$ dimensional filiform Lie algebras $(n \leq 11)$ from [4] we consider the structure constants $a_{i, j}^{k}$ in (3.1) to be fixed constants. 
Lemma 3.2. Let $L \in T$ Leib $_{n+1}$. Then

$$
\sum_{s=1}^{n-(i+j+k+1)} a_{j, k}^{s} b_{i, j+k+s}=\sum_{s=1}^{n-(i+j+k+1)}\left(a_{i, j}^{s} b_{i+j+s, k}-a_{i, k}^{s} b_{i+k+s, j}\right) .
$$

PRoof. The Leibniz identity for $e_{i}, e_{j}$ and $e_{k}$ gives the required relations between the structure constants

$$
\begin{aligned}
{\left[e_{i},\left[e_{j}, e_{k}\right]\right] } & =\left[e_{i}, \sum_{s=1}^{n-(j+k+1)} a_{j, k}^{s} e_{j+k+s}+b_{j, k} e_{n}\right] \\
& =\sum_{s=1}^{n-(i+j+k+1)} a_{j, k}^{s}\left(\sum_{t=1}^{n-(i+j+k+s+1)} a_{i, j+k+s}^{t} e_{i+j+k+s+t}+b_{i, j+k+s} e_{n}\right), \\
{\left[\left[e_{i}, e_{j}\right], e_{k}\right] } & =\left[\sum_{s=1}^{n-(i+j+1)} a_{i, j}^{s} e_{i+j+s}+b_{i, j} e_{n}, e_{k}\right] \\
& =\sum_{s=1}^{n-(i+j+k+1)} a_{i, j}^{s}\left(\sum_{t=1}^{n-(i+j+k+s+1)} a_{i+j+s, k}^{t} e_{i+j+k+s+t}+b_{i+j+s, k} e_{n}\right), \\
{\left.\left[e_{i}, e_{k}\right], e_{j}\right] } & =\left[\sum_{s=1}^{n-(i+k+1)} a_{i, k}^{s} e_{i+k+s}+b_{i, k} e_{n}, e_{j}\right] \\
& =\sum_{s=1}^{n-(i+j+k+1)} a_{i, k}^{s}\left(\sum_{t=1}^{n-(i+j+k+s+1)} a_{i+k+s, j}^{t} e_{i+j+k+s+t}+b_{i+k+s, j} e_{n}\right),
\end{aligned}
$$

leading to (3.4).

Here are several remarks regarding (3.4).

(1) It is symmetric with respect to $i, j, k$ (since $a_{s, t}^{k}=-a_{t, s}^{k}$ and $b_{s, t}=-b_{t, s}$ for any $s$ and $t$, except for $(s, t)=(0,0),(1,1),(0,1),(1,0))$.

(2) In the case where $(i, j, k)=(0, j, k)$ we get

$$
\sum_{s=1}^{n-(j+k+1)} a_{j, k}^{s} b_{0, j+k+s}=\sum_{s=1}^{n-(j+k+1)}\left(a_{0, j}^{s} b_{j+s, k}-a_{0, k}^{s} b_{k+s, j}\right),
$$

where $j \neq 0, k \neq 0$.

(3) Since $a_{0, t}^{s}=0$ as $s \neq 1$ and $a_{0, t}^{1}=-1$, we get

$$
a_{j, k}^{1} b_{0, j+k+1}+a_{j, k}^{2} b_{0, j+k+2}+\cdots+a_{j, k}^{n-(j+k+1)} b_{0, n-1}=-b_{j+1, k}+b_{k+1, j} .
$$

(4) Since $b_{0, t}=0$ as $t=2, \ldots, n-2$ and $b_{0, n-1}=-1$, we get that

$$
a_{j, k}^{n-(j+k+1)}=b_{j+1, k}-b_{k+1, j},
$$

for $k=j+1, j+2, \ldots, n-j-2$ and $j=1,2, \ldots,[(n-3) / 2]$. 
Lemma 3.3. Let $L \in T$ Leib $_{n+1}$. Then

$$
\left[e_{i}, e_{j+k}\right]=\sum_{s=0}^{k}(-1)^{k-s}\left(\begin{array}{l}
k \\
s
\end{array}\right)\left[e_{i+k-s}, e_{j}\right] R_{e_{0}}^{s},
$$

where $1 \leq i, j, k \leq n$ and $y R_{x}=[y, x]$ is the right multiplication operator on $L$.

PROOF. The proof will proceed by induction on $k$. Let $k=1$. Then $\left[e_{i}, e_{j+1}\right]=$ $\left[e_{i},\left[e_{j}, e_{0}\right]\right]=-\left[e_{i+1}, e_{j}\right]+\left[\left[e_{i}, e_{j}\right], e_{0}\right]$, that is, (3.5) holds for $k=1$. Then the following chain of equalities leads to the claim:

$$
\begin{aligned}
{\left[e_{i}, e_{j+k+1}\right]=} & {\left[e_{i},\left[e_{j+k}, e_{0}\right]\right]=\left[\left[e_{i}, e_{j+k}\right], e_{0}\right]-\left[\left[e_{i}, e_{0}\right], e_{j+k}\right] } \\
= & \sum_{s=0}^{k}(-1)^{k-s}\left(\begin{array}{l}
k \\
s
\end{array}\right)\left[e_{i+k-s}, e_{j}\right] R_{e_{0}}^{s+1} \\
& \quad-\sum_{s=0}^{k}(-1)^{k-s}\left(\begin{array}{l}
k \\
s
\end{array}\right)\left[e_{i+k+1-s}, e_{j}\right] R_{e_{0}}^{s} \\
= & -\sum_{s=1}^{k+1}(-1)^{k-s}\left(\begin{array}{c}
k \\
s-1
\end{array}\right)\left[e_{i+k+1-s}, e_{j}\right] R_{e_{0}}^{s} \\
& \quad-\sum_{s=0}^{k}(-1)^{k-s}\left(\begin{array}{c}
k \\
s
\end{array}\right)\left[e_{i+k+1-s}, e_{j}\right] R_{e_{0}}^{s} \\
= & \sum_{s=1}^{k}(-1)^{k+1-s}\left(\left(\begin{array}{c}
k \\
s-1
\end{array}\right)+\left(\begin{array}{c}
k \\
s
\end{array}\right)\right)\left[e_{i+k+1-s}, e_{j}\right] R_{e_{0}}^{s} \\
= & \sum_{s=0}^{k+1}(-1)^{k+1-s}\left(\begin{array}{c}
k+1 \\
s
\end{array}\right)\left[e_{i+k+1-s}, e_{j}\right] R_{e_{0}}^{s} .
\end{aligned}
$$

Let $L \in T$ Leib $_{n+1}$.

Definition 3.4. A basis $\left\{e_{0}, e_{1}, \ldots, e_{n}\right\}$ of $L$ is said to be adapted if its multiplication table with respect to $\left\{e_{0}, e_{1}, \ldots, e_{n}\right\}$ has the form of $T \operatorname{Leib}_{n+1}$.

Definition 3.5. Let $\left\{e_{0}, e_{1}, \ldots, e_{n}\right\}$ be an adapted basis of $L$. Then a nonsingular linear transformation $f: L \rightarrow L$ is said to be adapted if the basis $\left\{f\left(e_{0}\right), f\left(e_{1}\right), \ldots, f\left(e_{n}\right)\right\}$ is adapted.

To simplify notation, let

$$
a=\left(b_{0,0}, b_{0,1}, b_{1,1}, a_{i, j}^{1}, \ldots, a_{i, j}^{n-(i+j+1)}, b_{i, j}\right)
$$

stand for the algebra in $T$ Leib $_{n+1}$, with the structure constants $b_{0,0}, b_{0,1}, b_{1,1}, a_{i, j}^{1}, \ldots$, $a_{i, j}^{n-(i+j+1)}, b_{i, j}$. 
Let $a^{\prime}=\left(b_{0,0}^{\prime}, b_{0,1}^{\prime}, b_{1,1}^{\prime}, a_{i, j}^{1}, \ldots, a_{i, j}^{n-(i+j+1) \prime}, b_{i, j}^{\prime}\right)$. Consider an adapted transformation $f$. Let

$$
\begin{aligned}
& f\left(e_{0}\right)=A_{0} e_{0}+A_{1} e_{1}+\cdots+A_{n} e_{n}, \\
& f\left(e_{1}\right)=B_{0} e_{0}+B_{1} e_{1}+\cdots+B_{n} e_{n},
\end{aligned}
$$

where $A_{0} B_{1}-A_{1} B_{0} \neq 0$ and let $f(a)=a^{\prime}$.

LEMMA 3.4. For $b_{0,0}^{\prime}, b_{0,1}^{\prime}$ and $b_{1,1}^{\prime}$ the following hold:

$$
\begin{gathered}
b_{0,0}^{\prime}=\frac{A_{0}^{2} b_{0,0}+A_{0} A_{1} b_{0,1}+A_{1}^{2} b_{1,1}}{A_{0}^{n-1} B_{1}}, \quad B_{0}=0, \\
b_{1,1}^{\prime}=\frac{B_{1} b_{1,1}}{A_{0}^{n-1}}, \quad b_{0,1}^{\prime}=\frac{A_{0} b_{0,1}+2 A_{1} b_{1,1}}{A_{0}^{n-1}} .
\end{gathered}
$$

PROOF. By using multiplication on $a$ we get

$$
f\left(e_{i}\right)=\left[f\left(e_{i-1}\right), f\left(e_{0}\right)\right]=A_{0}^{i-2}\left(A_{0} B_{1}-A_{1} B_{0}\right) e_{i}+\sum_{s \geq i+1}(*) e_{s}, \quad 2 \leq i \leq n .
$$

Consider

$$
\left[f\left(e_{2}\right), f\left(e_{1}\right)\right]=a_{i, j}^{1 \prime} f\left(e_{4}\right)+\cdots+a_{i, j}^{(n-4) \prime} f\left(e_{n-1}\right)+b_{i, j}^{\prime} f\left(e_{n}\right) ;
$$

equating the corresponding coefficients, we get $B_{0}\left(A_{0} B_{1}-A_{1} B_{0}\right)=0$, which implies $B_{0}=0$.

The product $\left[f\left(e_{0}\right), f\left(e_{0}\right)\right]=b_{0,0}^{\prime} f\left(e_{n}\right)$ leads to

$$
A_{0}^{2} a_{0,0}+A_{0} A_{1} a_{0,1}+A_{1}^{2} a_{1,1}=a_{0,0}^{\prime} A_{0}^{n-1} B_{1} .
$$

Then we get

$$
b_{0,0}^{\prime}=\frac{A_{0}^{2} b_{0,0}+A_{0} A_{1} b_{0,1}+A_{1}^{2} b_{1,1}}{A_{0}^{n-1} B_{1}}
$$

Similarly,

$$
b_{1,1}^{\prime}=\frac{B_{1} b_{1,1}}{A_{0}^{n-1}}
$$

Now consider the equality

$$
b_{0,1}^{\prime} f\left(e_{n}\right)=\left[f\left(e_{1}\right), f\left(e_{0}\right)\right]+\left[f\left(e_{0}\right), f\left(e_{1}\right)\right] .
$$

Then $b_{0,1}^{\prime} A_{0}^{n-1} B_{1}=A_{0} B_{1} b_{0,1}+2 A_{1} B_{1} b_{1,1}$. This implies that

$$
b_{0,1}^{\prime}=\frac{A_{0} b_{0,1}+2 A_{1} b_{1,1}}{A_{0}^{n-1}} .
$$


Definition 3.6. The following adapted transformations of $L$ are said to be elementary:

$$
\begin{aligned}
\tau(a, b, c) & = \begin{cases}\tau\left(e_{0}\right)=a e_{0}+b e_{1}, \\
\tau\left(e_{1}\right)=c e_{1}, \\
\tau\left(e_{i+1}\right)=\left[\tau\left(e_{i}\right), \tau\left(e_{0}\right)\right], & 1 \leq i \leq n-1,\end{cases} \\
\sigma(a, k) & = \begin{cases}\sigma\left(e_{0}\right)=e_{0}+a e_{k}, \\
\sigma\left(e_{1}\right)=e_{1}, \\
\sigma\left(e_{i+1}\right)=\left[\sigma\left(e_{i}\right), \sigma\left(e_{0}\right)\right], & 1 \leq i \leq n-1,\end{cases} \\
\varphi(c, k) & = \begin{cases}\varphi\left(e_{0}\right)=e_{0}, \\
\varphi\left(e_{1}\right)=e_{1}+c e_{k}, \\
\varphi\left(e_{i+1}\right)=\left[\varphi\left(e_{i}\right), \varphi\left(e_{0}\right)\right], & 1 \leq i \leq n-1,\end{cases}
\end{aligned}
$$

where $a, b, c \in \mathbb{C}$; we refer to these as transformations of the first, second, and third type, respectively.

PROPOSITION 3.7. Let $f$ be an adapted transformation of L. Then it can be represented as the composition

$$
f=\varphi\left(B_{n}, n\right) \circ \cdots \circ \varphi\left(B_{2}, 2\right) \circ \sigma\left(A_{n}, n\right) \circ \cdots \circ \sigma\left(A_{2}, 2\right) \circ \tau\left(A_{0}, A_{1}, B_{1}\right) .
$$

PROOF. The proof is straightforward.

\section{The isomorphism criteria for truncated filiform Leibniz algebras}

In this section we consider a subclass of $T$ Leib $_{n+1}$. Let $L$ be an algebra from $T$ Leib $_{n+1}$ such that the quotient algebra $L /\left\langle e_{n}\right\rangle$ is isomorphic to an $n$-dimensional algebra from $N G F_{3}$. This kind of algebra we call truncated. Then it is easy to see that the multiplication table of $L$ has the form:

$$
\begin{cases}{\left[e_{i}, e_{0}\right]=e_{i+1},} & 1 \leq i \leq n-1, \\ {\left[e_{0}, e_{i}\right]=-e_{i+1},} & 2 \leq i \leq n-1, \\ {\left[e_{0}, e_{0}\right]=b_{0,0} e_{n},} & \\ {\left[e_{0}, e_{1}\right]=-e_{2}+b_{0,1} e_{n},} & \\ {\left[e_{1}, e_{1}\right]=b_{1,1} e_{n},} & 1 \leq i \leq n-1,1 \leq j \leq n-i, \\ {\left[e_{i}, e_{j}\right]=-\left[e_{j}, e_{i}\right]=b_{i, j} e_{n},} & 1 \leq i \leq n-1, \\ {\left[e_{i}, e_{n-i}\right]=-\left[e_{n-i}, e_{i}\right]=(-1)^{i} b_{i, n-i} e_{n},} & 1 \leq i \leq 1,\end{cases}
$$

where $b_{i, n-i}=b$ for $1 \leq i \leq n-1$, and $b \in\{0,1\}$ for odd $n$ and $b=0$ for even $n$. 
One of the reasons for considering the truncated case is to give a complete classification in this particular case and thus to show how the isomorphism criterion works in low-dimensional cases. Since in this case coefficients $a_{i, j}^{k}=0$, where $1 \leq i<j \leq n-1$ and $1 \leq k \leq n-(i+j+1)$, the multiplication table of $L$ has a simple form. Therefore the action of changing basis can easily be managed.

We now investigate the behavior of the coefficients $b_{i, j}$ under adapted basis changing. From Lemma 3.4 we conclude that the elementary transformation of the second and third types does not change the structure constants $b_{0,0}, b_{0,1}$ and $b_{1,1}$.

Consider

$$
\tau(a, b, c)= \begin{cases}\tau\left(e_{0}\right)=a e_{0}+b e_{1}, & \\ \tau\left(e_{1}\right)=c e_{1}, & a c \neq 0, \\ \tau\left(e_{i+1}\right)=\left[\tau\left(e_{i}\right), \tau\left(e_{0}\right)\right], & 1 \leq i \leq n-1 .\end{cases}
$$

We get

$$
\begin{aligned}
& \tau\left(e_{i}\right)=\left[\tau\left(e_{i-1)}, \tau\left(e_{0}\right)\right]=a^{i-1} c e_{i}+a^{i-2} b c b_{i-1,1} e_{n},\right. \\
& 2 \leq i \leq n-1, \tau\left(e_{n}\right)=a^{n-1} c e_{n} .
\end{aligned}
$$

The chain of equalities

$$
\begin{aligned}
a^{n-1} c b_{i, j}^{\prime} e_{n} & =b_{i, j}^{\prime} \tau\left(e_{n}\right)=\left[\tau\left(e_{i}\right), \tau\left(e_{j}\right)\right] \\
& =\left[a^{i-1} c e_{i}+a^{i-2} b c b_{i-1,1} e_{n}, a^{j-1} c e_{j}+a^{j-2} b c b_{j-1,1} e_{n}\right] \\
& =a^{i+j-2} c^{2}\left[e_{i}, e_{j}\right]=a^{i+j-2} c^{2} b_{i, j} e_{n},
\end{aligned}
$$

yields

$$
b_{i, j}^{\prime}=a^{i+j-1-n} c b_{i, j}
$$

Now consider

$$
\sigma(a, k)= \begin{cases}\sigma\left(e_{0}\right)=e_{0}+a e_{k}, & 2 \leq k \leq n-1, \\ \sigma\left(e_{1}\right)=e_{1}, & \\ \sigma\left(e_{i+1}\right)=\left[\sigma\left(e_{i}\right), \sigma\left(e_{0}\right)\right], & 1 \leq i \leq n-1 .\end{cases}
$$

Using induction it is easy to see that

$$
\sigma\left(e_{i}\right)=e_{i}+a b_{i-1, k} e_{n}, \quad 2 \leq i \leq n-1, \quad \sigma\left(e_{n}\right)=e_{n} .
$$

Then $\left[\sigma\left(e_{i}\right), \sigma\left(e_{j}\right)\right]=\left[e_{i}, e_{j}\right] . \quad$ So $b_{i, j}^{\prime}=b_{i, j}$, that is the elementary transformations of second type do not affect $b_{i, j}$.

LEMMA 4.1.

$$
b_{i+t, t}=(-1)^{s_{0}} b_{i+t-s_{0}, t+s_{0}}= \begin{cases}0, & i=2 s_{0}, \\ (-1)^{s_{0}} b_{i+t-s_{0}, t+s_{0},}, & i=2 s_{0}+1 .\end{cases}
$$

PROOF. Since $a_{i, j}^{k}=0$, due to (4) we get $b_{i+1, j}=-b_{i, j+1}, b_{j+2, j}=b_{j, j+2}=0$. A sequential application of this completes the proof. 
In Lemma 4.1, letting $t=1$ and $i=2 k$, we get $b_{2 k+1,1}=0$, and so $b_{1,2 k+1}=0$.

Consider the third type of transformation:

$$
\varphi\left(c_{k}, k\right)= \begin{cases}\varphi\left(e_{0}\right)=e_{0}, & 2 \leq k \leq n \\ \varphi\left(e_{1}\right)=e_{1}+c_{k} e_{k}, & 1 \leq i \leq n-1 \\ \varphi\left(e_{i+1}\right)=\left[\varphi\left(e_{i}\right), \varphi\left(e_{0}\right)\right], & \end{cases}
$$

Then $\varphi\left(e_{i}\right)=e_{i}+c_{k} e_{k+i-1}, 2 \leq i \leq n+1-k$, and $\varphi\left(e_{i}\right)=e_{i}, n+2-k \leq i \leq n$. Now the chain of equalities

$$
\begin{aligned}
b_{i, j}^{\prime} e_{n} & =b_{i, j}^{\prime} \varphi\left(e_{n}\right)=\left[\varphi\left(e_{i}\right), \varphi\left(e_{j}\right)\right]=\left[e_{i}+c_{k} e_{k+i-1}, e_{j}+c_{k} e_{k+j-1}\right] \\
& =\left[e_{i}, e_{j}\right]+c_{k}\left[e_{k+i-1}, e_{j}\right]+c_{k}\left[e_{i}, e_{k+j-1}\right]+c_{k}^{2}\left[e_{k+i-1}, e_{k+j-1}\right]
\end{aligned}
$$

yields

$$
\begin{aligned}
& b_{i, j}^{\prime}=b_{i, j}, \\
& b_{i, j}^{\prime}=b_{i, j}+c_{k}\left(b_{k+i-1, j}+b_{i, k+j-1}\right) \text {, } \\
& k+i+j-1 \geq n+1, \\
& k+i+j-1 \leq n, \\
& b_{i, j}^{\prime}=b_{i, j}+c_{k}\left(b_{k+i-1, j}+b_{i, k+j-1}\right)+c_{k}^{2} b_{k+i-1, k+j-1} \text {, } \\
& n+1 \leq 2 k+i+j-2 \text {, }
\end{aligned}
$$

Note that Lemma 4.1 implies the equalities

$$
b_{k+i-1, j}=(-1)^{k-1} b_{i, k+j-1} .
$$

As a result, for $b_{i, j}^{\prime}$ one has the following form:

$$
\begin{aligned}
& b_{i, j}^{\prime}=b_{i, j}, \quad n+2-(i+j) \leq k, \\
& b_{i, j}^{\prime}=b_{i, j}+c_{k}\left(1+(-1)^{k-1}\right) b_{i, j+k-1}, \\
& {\left[\frac{n+1-(i+j)}{2}\right]+1 \leq k \leq n+1-(i+j), } \\
& b_{i, j}^{\prime}=b_{i, j}+c_{k}\left(1+(-1)^{k-1}\right) b_{i, j+k-1} \\
&+c_{k}^{2}(-1)^{k-1} b_{i, j+2 k-2}, \quad k \leq\left[\frac{n-(i+j)}{2}\right]+1 .
\end{aligned}
$$

Applying sequentially the third type transformations $\varphi\left(c_{k}, k\right)$ for $k=2,3, \ldots, n$, we get the iteration

$$
b_{i, j}^{k}=b_{i, j}^{k-1}+c_{k}\left(1+(-1)^{k-1}\right) b_{i, j+k-1}^{k-1}+(-1)^{k-1} c_{k}^{2} b_{i, j+2 k-2}^{k-1},
$$

where the $b_{s, t}^{k-1}$ are supposed to be zero at $s+t \geq n+1$ and $b_{i, j}^{1}=b_{i, j}$.

Moreover, due to $b_{1,2 t+1}=0$ and $b_{i, j}=(-1)^{i-1} b_{1, i+j-1}$ (see Lemma 4.1), we can suppose, without loss of generality, that $n$ is even and the iteration can be reduced to

$$
\begin{gathered}
b_{1,2 i}^{k}=b_{1,2 i}^{k-1}+c_{k}\left(1+(-1)^{k-1}\right) b_{1,2 i+k-1}^{k-1}+(-1)^{k-1} c_{k}^{2} b_{1,2 i+2(k-1)}^{k-1}, \\
k \geq 2, i=1, \ldots, n / 2,
\end{gathered}
$$


where the $b_{1, t}^{k-1}$ are supposed to be zero at $t \geq n$ and $b_{1,2 i}^{1}=b_{1,2 i}$.

Here is a computer program in Maple which performs the above iterative algorithm.

Calling Sequence:

Iterate (Structure constants, Sequence, Dimension)

Parameters:

Structure constants - initial structure constants.

Sequence - sequence of elementary transformations.

Dimension - The dimension of the given algebra -1 .

$S C:=\operatorname{Vector}(n$, symbol $=b)$;

$\varphi:=\operatorname{Vector}(n$, symbol $=B)$;

for $i$ from 1 to $n$ do

if type $(i$, odd $)$ then $B_{i}:=0 \mathrm{fi}$;

if $i>n-2$ then $S C_{i}:=0 \mathrm{fi}$;

od;

Iterate $:=\operatorname{proc}(S C, \varphi, n)$

local $i$

for $i$ to trunc $(n / 2)$ do

if $2 i+k-1 \leq n$ then

$S C_{2 i}:=S C_{2 i}+B_{k}\left((-1)^{k-1}+1\right) S C_{2 i+k-1}$;

if $2 i+2 k-2 \leq n$ then

$S C_{2 i}:=S C_{2 i}+B_{k}^{2}(-1)^{k-1} S C_{2 i+2 k-2} ;$

fi;

fi;

od;

end proc;

for $k$ from 2 to $n$ do Iterate $(S C, \varphi, n)$ od;

$\operatorname{print}(S C)$;

4.1. Isomorphism criteria In this section, as an application of Sections 3 and 4, we give isomorphism criteria for the truncated filiform Leibniz algebras for some lowdimensional cases. Here those structure constants $b_{i, j}$ that are zero will be omitted.

(1) Two truncated filiform Leibniz algebras $a=\left(b_{0,0}, b_{0,1}, b_{1,1}, b_{1,2}\right)$ and $a^{\prime}=$ $\left(b_{0,0}^{\prime}, b_{0,1}^{\prime}, b_{1,1}^{\prime}, b_{1,2}^{\prime}\right)$ from $T$ Leib $_{5}$ are isomorphic if and only if there exist $A_{0}, A_{1}, B_{1} \in \mathbb{C}$ such that $A_{0} B_{1} \neq 0$ and the following equalities hold:

$$
\begin{gathered}
b_{0,0}^{\prime}=\frac{A_{0}^{2} b_{0,0}+A_{0} A_{1} b_{0,1}+A_{1}^{2} b_{1,1}}{A_{0}^{3} B_{1}}, \quad b_{1,1}^{\prime}=\frac{B_{1} b_{1,1}}{A_{0}^{3}}, \\
b_{0,1}^{\prime}=\frac{A_{0} b_{0,1}+2 A_{1} b_{1,1}}{A_{0}^{3}}, \quad b_{1,2}^{\prime}=\frac{B_{1}}{A_{0}^{2}} b_{1,2} .
\end{gathered}
$$

(2) Two truncated filiform Leibniz algebras $a=\left(b_{0,0}, b_{0,1}, b_{1,1}, b_{1,2}\right)$ and $a^{\prime}=$ $\left(b_{0,0}^{\prime}, b_{0,1}^{\prime}, b_{1,1}^{\prime}, b_{1,2}^{\prime}\right)$ from $T$ Leib $_{6}$ are isomorphic if and only if there exist 
$A_{0}, A_{1}, B_{1} \in \mathbb{C}$ such that $A_{0} B_{1} \neq 0$ and the following equalities hold:

$$
\begin{gathered}
b_{0,0}^{\prime}=\frac{A_{0}^{2} b_{0,0}+A_{0} A_{1} b_{0,1}+A_{1}^{2} b_{1,1}}{A_{0}^{4} B_{1}}, \quad b_{1,1}^{\prime}=\frac{B_{1} b_{1,1}}{A_{0}^{4}}, \\
b_{0,1}^{\prime}=\frac{A_{0} b_{0,1}+2 A_{1} b_{1,1}}{A_{0}^{4}}, \quad b_{1,2}^{\prime}=\frac{B_{1}}{A_{0}^{3}} b_{1,2} .
\end{gathered}
$$

(3) Two truncated filiform Leibniz algebras $a=\left(b_{0,0}, b_{0,1}, b_{1,1}, b_{1,2}, b_{1,4}\right)$ and $a^{\prime}=\left(b_{0,0}^{\prime}, b_{0,1}^{\prime}, b_{1,1}^{\prime}, b_{1,2}^{\prime}, b_{1,4}^{\prime}\right)$ from $T \mathrm{Leib}_{7}$ are isomorphic if and only if there exist $A_{0}, A_{1}, B_{1} \in \mathbb{C}$ such that $A_{0} B_{1} \neq 0$ and the following equalities hold:

$$
\begin{gathered}
b_{0,0}^{\prime}=\frac{A_{0}^{2} b_{0,0}+A_{0} A_{1} b_{0,1}+A_{1}^{2} b_{1,1}}{A_{0}^{5} B_{1}}, \quad b_{1,1}^{\prime}=\frac{B_{1} b_{1,1}}{A_{0}^{5}}, \\
b_{0,1}^{\prime}=\frac{A_{0} b_{0,1}+2 A_{1} b_{1,1}}{A_{0}^{5}}, \quad b_{1,2}^{\prime}=\frac{B_{1}}{A_{0}^{4}}\left(b_{1,2}-\left(B_{2}^{2}-2 B_{3}\right)\right) b_{1,4}, \quad b_{1,4}^{\prime}=\frac{B_{1}}{A_{0}^{4}} b_{1,4} .
\end{gathered}
$$

(4) Two truncated filiform Leibniz algebras $a=\left(b_{0,0}, b_{0,1}, b_{1,1}, b_{1,2}, b_{1,4}\right)$ and $a^{\prime}=\left(b_{0,0}^{\prime}, b_{0,1}^{\prime}, b_{1,1}^{\prime}, b_{1,2}^{\prime}, b_{1,4}^{\prime}\right)$ from $T$ Leib $_{8}$ are isomorphic if and only if there exist $A_{0}, A_{1}, B_{1} \in \mathbb{C}$ such that $A_{0} B_{1} \neq 0$ and the following equalities hold:

$$
\begin{gathered}
b_{0,0}^{\prime}=\frac{A_{0}^{2} b_{0,0}+A_{0} A_{1} b_{0,1}+A_{1}^{2} b_{1,1}}{A_{0}^{6} B_{1}}, \quad b_{1,1}^{\prime}=\frac{B_{1} b_{1,1}}{A_{0}^{6}}, \\
b_{0,1}^{\prime}=\frac{A_{0} b_{0,1}+2 A_{1} b_{1,1}}{A_{0}^{6}}, \quad b_{1,2}^{\prime}=\frac{B_{1}}{A_{0}^{5}}\left(b_{1,2}-\left(B_{2}^{2}-2 B_{3}\right)\right) b_{1,4}, \quad b_{1,4}^{\prime}=\frac{B_{1}}{A_{0}^{5}} b_{1,4} .
\end{gathered}
$$

(5) Two truncated filiform Leibniz algebras $a=\left(b_{0,0}, b_{0,1}, b_{1,1}, b_{1,2}, b_{1,4}, b_{1,6}\right)$ and $a^{\prime}=\left(b_{0,0}^{\prime}, b_{0,1}^{\prime}, b_{1,1}^{\prime}, b_{1,2}^{\prime}, b_{1,4}^{\prime}, b_{1,6}^{\prime}\right)$ from $T$ Leib 9 are isomorphic if and only if there exist $A_{0}, A_{1}, B_{1} \in \mathbb{C}$ such that $A_{0} B_{1} \neq 0$ and the following equalities hold:

$$
\begin{gathered}
b_{0,0}^{\prime}=\frac{A_{0}^{2} b_{0,0}+A_{0} A_{1} b_{0,1}+A_{1}^{2} b_{1,1}}{A_{0}^{7} B_{1}}, \quad b_{1,1}^{\prime}=\frac{B_{1} b_{1,1}}{A_{0}^{7}}, \\
b_{0,1}^{\prime}=\frac{A_{0} b_{0,1}+2 A_{1} b_{1,1}}{A_{0}^{7}}, \\
b_{1,2}^{\prime}=\frac{B_{1}}{A_{0}^{6}}\left(b_{1,2}-\left(B_{2}^{2}-2 B_{3}\right) b_{1,4}+\left(B_{3}^{2}-2 B_{2}^{2} B_{3}+2 B_{5}\right) b_{1,6}\right), \\
b_{1,4}^{\prime}=\frac{B_{1}}{A_{0}^{6}}\left(b_{1,4}-\left(B_{2}^{2}-2 B_{3}\right)\right) b_{1,6}, \quad b_{1,6}^{\prime}=\frac{B_{1}}{A_{0}^{6}} b_{1,6} .
\end{gathered}
$$

\section{Acknowledgements}

The authors thank Sh. I. Rakhimov for writing the Maple program in Section 4. We are grateful to the referee for helpful comments. 


\title{
References
}

[1] Sh. A. Ayupov and B. A. Omirov, 'On some classes of nilpotent Leibniz algebras', Siberian Math. J. (1) 42 (2001), 18-29

[2] U. D. Bekbaev and I. S. Rakhimov, On classification of finite dimensional complex filiform Leibniz algebras (part 1), http://front.math.ucdavis.edu/, ArXiv:math. RA/01612805, 2006.

[3] - On classification of finite dimensional complex filiform Leibniz algebras (part 2), http://front.math.ucdavis.edu/, ArXiv:0704.3885v1 [math.RA], 30 Apr 2007.

[4] J. R. Gómez, A. Jimenéz-Merchán and Y. Khakimdjanov, 'Low-dimensional filiform Lie algebras', J. Pure Appl. Algebra 130 (1998), 133-158

[5] J. R. Gómez and B. A. Omirov, On classification of complex filiform Leibniz algebras, arXive:math/0612735 v1 [math.R.A.], 23 Dec 2006.

[6] M. Goze and Yu. Khakimjanov, Nilpotent Lie Algebras, Mathematics and its Applications, 361 (Kluwer Academic Publishers, Dordrecht, 1996), p. 336

[7] Y. Kosman-Schwarzbach, 'From Poisson algebras to Gerstenhaber algebras', Ann. Inst. Fourier (Grenoble) 46 (1996), 1243-1274

[8] J.-L. Loday, 'Une version non commutative des algèbres de Lie: les algèbres de Leibniz', L'Ens. Math. 39 (1993), 269-293

[9] J.-L. Loday and T. Pirashvili, 'Universal enveloping algebras of Leibniz algebras and (co)homology', Math. Ann. 296 (1993), 139-158

[10] I. S. Rakimov and S. K. Said Husain, On isomorphism classes and invariants of low-dimensional complex filiform Leibniz algebras (Part 1), http://front.math.ucdavis.edu/, ArXiv:0710.0121 v1.[math RA], 1 Oct 2007.

[11] - On isomorphism classes and invariants of low-dimensional complex filiform Leibniz algebras (Part 2), http://front.math.ucdavis.edu/, ArXiv:0806.1803 v1.[math RA], 11 June 2008.

[12] M. Vergne, 'Cohomologie des algèbres de Lie nilpotentes. Application à l'étude de la variété des algèbres de Lie nilpotentes', Bull. Soc. Math. France 98 (1970), 81-116

\section{B. A. OMIROV, Institute of Mathematics, Uzbekistan Academy of Science, F. Hodjaev str. 29, 100125 Tashkent, Uzbekistan \\ e-mail: omirovb@mail.ru}

\author{
I. S. RAKHIMOV, Institute for Mathematical Research (INSPEM), \\ Department of Mathematics, Universiti Putra Malaysia, Faculty of Science, \\ 43400 UPM Serdang, Selangor Darul Ehsan, Malaysia \\ e-mail: isamiddin@science.upm.edu.my, risamiddin@mail.ru
}

